\title{
STATIONARY LOGIC AND ORDINALS ${ }^{1}$
}

BY

D. G. SEESE

\begin{abstract}
The $L$ (aa)-theory of ordinals is investigated. It is proved that this theory is decidable and that each ordinal is finitely determinate.
\end{abstract}

0. Introduction. The quantifier "aa $X$ " with the intended meaning "there is a closed and unbounded system of countable sets $X$ " was introduced by Shelah in [25]. If we enrich the logic $L_{\omega \omega}$ by this quantifier we get the stationary logic $L_{\omega \omega}(\mathbf{a a})$. The fundamental paper for the study of stationary logic is [1]. Stationary logic has a smooth model theory and is a generalization of the logic $L_{\omega \omega}\left(Q_{1}\right)$ with the quantifier "there exist uncountably many". But unfortunately $L_{\omega \omega}$ (aa) does not possess all good properties of $L_{\omega \omega}\left(Q_{1}\right)$. For instance $L_{\omega \omega}(\mathbf{a a})$-elementary equivalence is not preserved by performing disjoint unions and finite direct products. Moreover Harrington, Kunen and Shelah proved that the existence of $L$ (aa)-elementary substructures of cardinality $\omega_{1}$ of all uncountable structures is independent of ZFC (given the consistency of the existence of a super compact cardinal). The reasons for this negative behaviour of stationary logic are the properties of the dual quantifier “ $\neg \mathbf{a a} X \neg$ ” denoted by "stat $X$ ”. To avoid these difficulties Kaufmann introduced in his thesis [10] (see also [1]) the notion of finitely determinate structures. A structure is said to be finitely determinate if it satisfies all instances of the scheme

$$
\text { aa } \vec{X} \forall \vec{x}[\text { aa } Y \varphi(\vec{X}, \vec{x}, Y) \vee \text { aa } Y \neg \varphi(\vec{X}, \vec{x}, Y)]
$$

where $\varphi$ ranges over formulas in $L(\mathbf{a a})$. Moreover Kaufmann [10] developed a useful back-and-forth criterion with the help of which it is possible to show that two structures are $L($ aa)-elementary equivalent as well as finitely determinate. Using this criterion Kaufmann proved that $L(\mathbf{a a})$-elementary equivalence is preserved for finitely determinate structures by forming disjoint unions and finite direct products. He proved that all ordinals $<\omega_{1}^{\omega_{1}}$ are finitely determinate. Kaufmann raised the problem whether all ordinals are finitely determinate. This problem was left open also in [7] where a deep investigation of finitely determinate structures is presented.

In $\S 2$ of this article it is proved that infinite ordered sums of finitely determinate structures are finitely determinate if all components are $L(\mathbf{a a})$-equivalent. Moreover

Received by the editors June 20, 1979 and, in revised form, December 14, 1979.

AMS (MOS) subject classifications (1970). Primary 02B20, $02 \mathrm{G} 05$.

Key words and phrases. Stationary logic, finitely determinate structure, ordinal, linear ordering.

${ }^{1}$ During the preparation of the first version of this article the author was a guest of the Academy of Science of the USSR in Novosibirsk. 
it is proved that $L(\mathbf{a a})$-elementary equivalence is preserved by performation of such ordered sums.

This leads in $\$ 3$ to a positive answer to the above question of Kaufmann [10], i.e. all ordinals are finitely determinate. Let $K$ be the smallest class of ordinals which contains $1, \omega$ and $\omega_{1}$ and is closed by the operations $\alpha+\beta$ and $\alpha \cdot \beta$ (for $\alpha$, $\beta \in K$ ). It is proved that the (aa)-theory of the class of all ordinals is just the (aa)-theory of $K$ and that this theory is decidable.

The proof of Theorem 3.3 in an earlier version of this article was incorrect. This was pointed out to me by $H$. Herre [8] and the referee. The referee sent me a correct proof of this result. He generalized also an earlier version of Theorem 2.1. I am very grateful to the referee for the permission to use his proofs of Theorem 2.1 and Theorem 3.3 and for several other suggestions and corrections.

1. Preliminaries. Throughout this paper $L$ denotes a finite elementary language. Structures for $L$ are denoted by $\mathfrak{A}, \mathfrak{B}$, etc. and their universes $|\mathfrak{A}|,|\mathfrak{B}|$, etc. by the corresponding capital letters $A, B$, etc. For a set $M$ let $P_{\omega_{1}}(M)$ denote the set of all countable subsets of $M$. Closed and unbounded (cub) subsets of $P_{\omega_{1}}(A), P_{\omega_{1}}(B)$, etc. are denoted by $\mathscr{Q}, \mathscr{B}$, etc. If $L^{\prime}$ is an extension of $L$ by a relation symbol $R$ and $\mathfrak{A}$ is a structure for $L^{\prime}$ then the interpretation of $R$ in $\mathfrak{A}$ is denoted by $R(\mathfrak{U})$ or sometimes by $R_{\mathfrak{X}}$.

To get $L\left(\right.$ aa) we expand $L$ by adding countably many set variables $X_{1}, X_{2}, \ldots$, the $\in$ symbol and a new quantifier aa. Formulas of $L(\mathbf{a a})$ are formed as usual with the new formation rule:

If $\varphi$ is a formula of $L(\mathbf{a a})$ so is $(\operatorname{aa} X) \varphi$ for each set variable $X$. For an $L$ structure $\mathfrak{A}, \mathfrak{A} \vDash(\operatorname{aa} X) \varphi(X)$ holds iff there is a cub collection $Q \subseteq P_{\omega_{1}}(A)$ such that, for all $B \in \mathbb{Q}, \mathfrak{A} \vDash \varphi[B]$ holds.

Let $K$ be a class of structures for $L$; then $\operatorname{Th}_{L(\text { an })}(K), \operatorname{Th}_{L\left(Q_{1}\right)}(K), \operatorname{Th}_{L}(K)$ denote the theory of $K$ in the language $L(\mathbf{a a}), L\left(Q_{1}\right), L$ respectively. If the language $L$ is fixed then we denote these theories by $\mathrm{Th}_{\mathrm{ag}}(K), \mathrm{Th}_{Q_{1}}(K), \operatorname{Th}(K)$ respectively. In the case that $K$ has only one element $\mathfrak{A}$ we write $\operatorname{Th}_{L(\mathfrak{a a})}(\mathfrak{U}), \operatorname{Th}_{L\left(Q_{1}\right)}(\mathfrak{U}), \operatorname{Th}_{L}(\mathfrak{A})$ respectively.

By induction on the complexity of $\varphi$ we define the quantifier rank of $\varphi$.

$$
\begin{aligned}
\operatorname{qr} \varphi & :=0 \quad \text { if } \varphi \text { is atomic, } \\
\operatorname{qr} \varphi \wedge \psi & :=\max (\{\operatorname{qr} \varphi, \operatorname{qr} \psi\}), \quad \operatorname{qr} \neg \varphi:=\operatorname{qr} \varphi, \\
\operatorname{qr}(\operatorname{aa} X) \varphi & :=\operatorname{qr}(\exists x) \varphi:=\operatorname{qr} \varphi+1 .
\end{aligned}
$$

For two $L$ structures $\mathfrak{A}$ and $\mathfrak{B}$ and a natural number $n$ we write

$\mathfrak{A} \equiv_{n} \mathfrak{B}(L($ aa $))$ iff for all sentences $\varphi$ of $L($ aa) with qr $\varphi \leqslant n: \mathfrak{A} \vDash \varphi$ iff $\mathfrak{B} \vDash \varphi$.

In this case $\mathfrak{A}$ and $\mathfrak{B}$ are said to be ( $L(\mathrm{aa}), n$ )-equivalent. Sometimes we use these notations without mentioning the language $L$.

The following convention we take from [10]. Suppose that $\mathfrak{A}$ and $\mathfrak{B}$ are $L$ structures and that $f$ is a partial function from $A$ to $B$. We write " $\mathscr{A} \vDash \varphi(\operatorname{dom} f)$ iff $\mathfrak{B} \vDash \varphi(\mathrm{rn} f)$ " as abbreviation for the following statement: For any assignment $g$ from the free variables $\left(x_{1}, \ldots, x_{n}\right)$ of $\varphi$ to $A$, if the range of $g$ is contained in the 
domain of $f$, then

$$
\mathfrak{A} \vDash \varphi\left[g\left(x_{1}\right), \ldots, g\left(x_{n}\right)\right] \quad \text { iff } \quad \mathfrak{B} \vDash \varphi\left[\left(f\left(g\left(x_{1}\right)\right), \ldots, f\left(g\left(x_{n}\right)\right)\right)\right] .
$$

This convention also extends in the obvious way to $L(\mathbf{a a})$, where $f \subseteq[A \times B] \cup$ $\left[P_{\omega_{1}}(A) \times P_{\omega_{1}}(B)\right]$. “( $(\mathfrak{A}, \mathrm{rn} f) \vDash \varphi$ iff $(\mathfrak{B}, \operatorname{dom} f) \vDash \varphi$ " is used in a similar way.

For any sets $A$ and $B$ write $A * B$ for $[A \times B] \cup\left[P_{\omega_{1}}(A) \times P_{\omega_{1}}(B)\right] . \vec{X}, \vec{x}, \vec{a}, \vec{A}$ denote finite sequences of set variables, individual variables, individual constants and set constants respectively. Sometimes it is necessary to use $\vec{a}, \vec{b}, \vec{c}$ also to denote finite sequences of elements from $M \cup P_{\omega_{1}}(M)$ for a certain set $M$. This will be mentioned at the corresponding place.

Sometimes the language $L$ for linear orderings will be extended by other predicates or constants. The corresponding models for these languages will be denoted as linear orderings if their reducts to $L$ are linear orderings.

Let $L_{1}, L_{2}$ be extensions of the language $L$ for linear orderings by a finite number of relation symbols. Moreover let $\mathfrak{A}$ be a linear ordering for $L_{1}$. For each $a \in A$ let $\mathfrak{S}_{a}$ be a linear ordering for $L_{2}$. The ordered sum $\mathfrak{E}=\Sigma_{\mathscr{Y}} \mathcal{E}_{a}$ is defined as follows.

The domain of $\mathbb{E}$ is the disjoint union $\dot{\cup}_{a \in A} C_{a}$.

$$
<_{\S}:=\bigcup_{a \in A}<_{\S_{a}} \bigcup\{(c, d): \text { there are } a, b \in A
$$

$$
\text { with } \left.a<_{\mathfrak{A}} b \& c \in C_{a} \& d \in C_{b}\right\} \text {. }
$$

For each $n$-ary $P$ in the language of $\mathfrak{A}$,

$$
P(\mathfrak{c}):=\left\{\left(b_{1}, \ldots, b_{n}\right): \text { for some }\left(a_{1}, \ldots, a_{n}\right) \in P(\mathfrak{A}), b_{i} \in C_{a_{i}}(1 \leqslant i<n)\right\} .
$$

For each $n$-ary $S$ in the language of $\mathfrak{\mho}_{a}, S(\mathbb{E}):=\dot{U}_{a \in A} S\left(\aleph_{a}\right)$. For a linear ordering $\mathfrak{A}$ and an element $a \in A$ we denote the structure $(\{c: c<a \& c \in A\}$, $<\uparrow\{c: c<a \& c \in A\})$ as initial segment of $\mathfrak{A}$ generated by $a$. If $a$ and $b$ are elements of $A$ then the structure $(\{c: a \leqslant c \leqslant b\},<\uparrow\{c: a \leqslant c \leqslant b\})$ is denoted as closed interval between $a$ and $b$. The cofinality of a linear ordering we define in the same way as for ordinals.

Let $\alpha$ be an ordinal. Then the structure $(\alpha,<)$, where $<$ is the usual $\in$ relation will be denoted also by $\alpha$. Hence we can use e.g. $\alpha \vDash \varphi$ or $\alpha \equiv_{n} \beta(L(\mathbf{a a}))$ instead of $(\alpha,<) \vDash \varphi$ or $(\alpha,<) \equiv_{n}(\beta,<)(L(\mathbf{a a}))$ respectively.

If all linear orderings $\mathbb{E}_{a}(a \in A)$ are isomorphic to a linear ordering $\sqrt{5}$ then we write $\Sigma_{\mathfrak{A}} \mathfrak{E}$ or $\mathfrak{E} \cdot \mathfrak{A}$ instead of $\Sigma_{\mathfrak{A}} \mathfrak{E}_{a}$. Throughout this paper we shall use ZFC as metatheory.

Following Kaufmann [10] we define for an elementary language $L$, a natural number $k$ and an $L$ structure $\mathfrak{A}$ :

$\mathfrak{A}$ is finitely $k$-determinate if every sentence of $L(\mathbf{a a})$ which has the following form, and has quantifier rank at most $k$, is true in $\mathfrak{A}$ :

$$
(\mathbf{a a} \vec{X})(\forall \vec{x})[(\operatorname{stat} Y) \psi(\vec{X}, Y, \vec{x}) \leftrightarrow(\mathbf{a a} Y) \psi(\vec{X}, Y, \vec{x})]
$$

$\mathfrak{A}$ is finitely determinate if $\mathfrak{A}$ is finitely $k$-determinate for each $k<\omega$.

Finitely determinate structures have nice model theoretic properties. A lot of results about finitely determinate structures can be found in [10], [7], [1]. 
Let $S_{1}$ be a stationary subset of $\omega_{1}$ such that also the complement $\bar{S}_{1}$ of $S_{1}$ is stationary. Then $\left(\omega_{1},<, S_{1}\right)$ is not finitely determinate. A proof of this can be found in [10]. From this example it is easy to find a linear ordering and a Boolean algebra which is not finitely determinate (see [7]). This example led to the following result.

Theorem 1.1 (Seese, Tuschik, Weese [23]). Let LO, BA denote the class of linear orderings, Boolean algebras respectively. Then $\operatorname{Th}_{\mathrm{aa}}(L O)$ and $\operatorname{Th}_{\mathrm{aa}}(B A)$ are undecidable.

A proof of this theorem can be found in [23]. The following result is related to the first part of Theorem 1.1. The proof is similar to the proof of Theorem 1.1 in [23].

THEOREM 1.2. Let $K$ be the following class of structures.

$$
K:=\left\{\left(\left(\omega_{1} \cdot \omega^{2}\right),<, S\right): S \subseteq\left(\omega_{1} \cdot \omega^{2}\right)\right\} .
$$

Then $\mathrm{Th}_{\mathrm{ag}}(K)$ is undecidable.

Proof. Let $K^{\prime}$ be $\left\{\left(\left(\omega_{1} \cdot \omega^{2}\right),<, S_{1}, S_{2}\right): S_{1}, S_{2} \subseteq\left(\omega_{1} \cdot \omega^{2}\right)\right\}$. For each $\mathfrak{A}=\left(\left(\omega_{1} \cdot\right.\right.$ $\left.\left.\omega^{2}\right),<, S\right) \in K$ let $\mathfrak{A}^{\prime}$ be the following structure:

$$
\begin{aligned}
\mathfrak{U}^{\prime} & :=\left\{a: a \in\left(\omega_{1} \cdot \omega^{2}\right) \& a \text { is a limit ordinal }\right\}, \\
<_{\mathfrak{H}^{\prime}} & :=<_{\mathfrak{A}} \uparrow A^{\prime}, \\
S_{1}\left(\mathfrak{U}^{\prime}\right) & :=\left\{a: a \in A^{\prime} \& a+1 \in S\right\}, \\
S_{2}\left(\mathfrak{U}^{\prime}\right) & :=\left\{a: a \in A^{\prime} \& a+2 \in S\right\} .
\end{aligned}
$$

Obviously $\mathfrak{U}^{\prime} \in K^{\prime}$ and for each $\mathfrak{B} \in K^{\prime}$ there exists a structure $\mathbb{E} \in K$ with $\mathfrak{E}^{\prime} \cong \mathfrak{B}$.

Using these facts and the well-known interpretability technique (which can be extended in this case to stationary logic) we get the interpretability of $\operatorname{Th}_{\mathrm{aa}}\left(K^{\prime}\right)$ in $\mathrm{Th}_{\mathrm{ag}}(K)$.

Now we prove the interpretability of the elementary theory of one binary irreflexive and symmetric relation in $\operatorname{Th}_{a \mathrm{ag}}\left(K^{\prime}\right)$. This is sufficient since this theory is undecidable (see [19]). At first we have to remember that there are $\omega_{1}$ many pairwise disjoint stationary subsets of $\omega_{1}$ (see [11], [29]). Let $A_{\nu}\left(\nu \in \omega_{1}\right)$ be such sets.

Now let $(A, R)$ be an at most countable irreflexive and symmetric graph. Let $B$ be the set of all elements of $\omega_{1} \cdot \omega^{2}$ of the form $\left(\omega_{1} \cdot \omega\right) \cdot n$ (for $n \in \omega$ ) and let $f$ be a 1-1 embedding of $A$ into $B$. We define $S_{1}$ to be the range of $f$. For each element $b$ of $B$ we define $s c_{0}(b)$ to be the smallest element $c$ of $B$ with $b<c$. Similarly for each $c \in\left|\omega_{1} \cdot \omega^{2}\right|, s c_{1}(c)$ denotes the smallest element $d$ of $\left|\omega_{1} \cdot \omega^{2}\right|$ with $c<d$ and $c f(d)=\omega_{1}$. If $c, d$ are points of $\omega_{1} \cdot \omega^{2}$ then $\left[c_{1}, c_{2}\right)$ denotes the restriction of $\omega_{1} \cdot \omega^{2}$ to $\left\{d: d \in\left|\omega_{1} \cdot \omega^{2}\right| \& c_{1} \leqslant d<c_{2}\right\}$. For each subset $S$ of $\omega_{1} \cdot \omega^{2}, S \uparrow\left[c_{1}, c_{2}\right)$ denotes $\left\{d: d \in\left|\omega_{1} \cdot \omega^{2}\right| \& c_{1} \leqslant d<c_{2}\right\} \cap S$.

Now we can define $S_{2}$ in such a way that the following conditions are fulfilled: 
(1) For all $a \in\left|\omega_{1} \cdot \omega^{2}\right|$ with $c f(a)=\omega_{1}$ there is a $j \in \omega$ such that $S_{2} \uparrow\left[a, s c_{1}(a)\right)$ $=\varnothing$ or

$$
\left(\left[a, s c_{1}(a)\right), S_{2} \uparrow\left[a, s c_{1}(a)\right)\right) \cong\left(\omega_{1}, A_{j}\right) .
$$

(2) For all $a, b \in A$ we have $(a, b) \in R$ iff $a \neq b$ and there are elements $c_{1}, c_{2}$ from $\omega_{1} \cdot \omega^{2}$ such that

$$
\begin{gathered}
c f\left(c_{1}\right)=c f\left(c_{2}\right)=\omega_{1}, \quad f(a) \leqslant c_{1}<s c_{0}(f(a)), \\
f(b) \leqslant c_{2}<s c_{0}(f(b)) \quad \text { and } \\
\left(\left[c_{1}, s c_{1}\left(c_{1}\right)\right), S_{2} \uparrow\left[c_{1}, s c_{1}\left(c_{1}\right)\right)\right) \cong\left(\left[c_{2}, s c_{1}\left(c_{2}\right)\right), S_{2} \uparrow\left[c_{2}, s c_{1}\left(c_{2}\right)\right)\right) .
\end{gathered}
$$

This is possible since $A$ is at most countable. But using an idea of Shelah $(+)$ can be expressed in $L^{\prime}\left(\right.$ aa) where $L^{\prime}$ is the language for $\left(\omega_{1} \cdot \omega^{2},<, S_{1}, S_{2}\right)$ (see [10, Example 2.6]). This proves the undecidability of $\mathrm{Th}_{a \mathrm{a}}(K)$. Q.E.D.

Theorem 1.1 is a correction of [21] where the opposite was announced. It answers a corresponding question from [7].

Eklof and Mekler proved in [7] that Abelian groups and moreover modules are finitely determinate. There and in [2] is also proved that the (aa)-theory of Abelian groups is decidable. In [22] it is proved that each well-founded tree of height $\leqslant \omega$ is finitely determinate and that the corresponding (aa)-theory is decidable. Barwise and Kunen showed that $\omega_{1}$ is finitely determinate (see [10] for a proof). Kaufmann (see [7]) proved also that $1+\left(\eta \cdot \omega_{1}\right)$ and $(1+\eta) \cdot \omega_{1}$ are finitely determinate. Moreover he proved the following results.

Lemma 1.3 (KaUfMANN [10]). Suppose $\left(\mu_{n}: n<\omega\right)$ is a countable sequence of finitely determinate ordinals and set $\mu:=\cup_{n<\omega} \mu_{n}$. Then $\mu$ is finitely determinate.

LEMma 1.4 (KAUfMANN [10]). If $\alpha$ and $\beta$ are finitely determinate ordinals, so are $\alpha+\beta$ and $\alpha \cdot \beta$.

TheOREM 1.5 (KAUfMANN [10]). The class $X$ of finitely determinate ordinals has the following properties.

(i) $X$ is an initial segment of the ordinals (under $\epsilon$ ).

(ii) $X$ has uncountable cofinality (if some ordinal is not in $X$ ).

(iii) $\omega_{1} \in X$.

Hence by the results of Kaufmann each ordinal $<\omega_{1}^{\omega_{1}}$ is finitely determinate.

Caicedo [6], Kaufmann [10], Makowsky [14] and Weese and the author [24] developed back-and-forth criteria to establish ( $L(\mathbf{a a}), n)$-equivalence. A detailed investigation can be found in [10]. There also determinate back-and-forth systems are introduced which are a useful tool to investigate finitely determinate structures. The following definition we take from [10].

Definition 1.6 (Kaufmann [10]). Fix two L structures $\mathfrak{A}$ and $\mathfrak{B} .\left(F_{k}: k \leqslant n\right)$ is a determinate $(L(\mathbf{a a}), n)$ back-and-forth system from $\mathfrak{A}$ to $\mathfrak{B}$ if it satisfies the following conditions:

(1) For all $f \in F_{0}, f \subseteq A * B$ and for every atomic formula $\varphi, \mathfrak{A} \vDash \varphi(\operatorname{dom} f)$ iff $\mathfrak{B} \vDash \varphi(\mathrm{rn} f)$.

(2) $0 \in F_{n}$. 
(3) Whenever $k<l \leqslant n$ and $f \in F_{l}$ :

(i) $(\forall a \in A)(\exists b \in B)\left[f \cup\{(a, b)\} \in F_{k}\right]$,

(ii) $(\forall b \in B)(\exists a \in A)\left[f \cup\{(a, b)\} \in F_{k}\right]$,

(iii) there are cub collections $\varrho \subseteq P_{\omega_{1}}(A)$ and $\mathscr{D} \subseteq P_{\omega_{1}}(B)$ such that

$$
(\forall C \in \mathcal{C})(\forall D \in \mathscr{D})\left[f \cup\{(C, D)\} \in F_{k}\right] \text {. }
$$

TheOREM 1.7 (KAUfMANN [10]). If $L$ is finite, $\mathfrak{A}$ and $\mathfrak{B}$ are $L$ structures and $n$ is $a$ natural number, then the following are equivalent.

(i) There is a determinate ( $L(\mathbf{a a}), n)$ back-and-forth system from $\mathfrak{A}$ to $\mathfrak{B}$.

(ii) $\mathfrak{A} \equiv_{n} \mathfrak{B}(L(\mathrm{aa}))$ and $\mathfrak{A}$ is (and $\mathfrak{B}$ is) finitely $n$-determinate.

Sometimes also the following simple and more technical result is useful.

LEMMA 1.8. Let $\mathfrak{A}$ and $\mathfrak{B}$ be two $L$ structures for a finite language $L$ and let $n$ be $a$ natural number. Moreover let (i) and (ii) be fulfilled.

(i) $\mathfrak{A} \equiv_{n} \mathfrak{B}(L(\mathbf{a a}))$.

(ii) There exists a cub system $\bigodot$ for $A$ such that

$$
(\forall C \in \mathcal{C})(\forall D \in \mathcal{C})(\mathfrak{A}, C) \equiv_{n-1}(\mathfrak{A}, D)(L(\mathbf{a a})) .
$$

Then there exists a cub system $\mathscr{D}$ for $B$ such that

$$
(\forall C \in \mathcal{C})(\forall D \in \mathscr{D})(\mathfrak{A}, C) \equiv_{n-1}(\mathfrak{B}, D)(L(\mathbf{a a})) \text {. }
$$

Proof. Let $C_{0} \in \mathcal{C}$. Since $L$ is a finite language there is a formula $\varphi(X)$ of $L($ aa) such that for all $C \in P_{\omega_{1}}(A)$ (for all $\left.D \in P_{\omega_{1}}(B)\right)(\mathfrak{A}, C) \vDash \varphi[C]$ iff $(\mathfrak{A}, C$ ) $\equiv_{n-1}\left(\mathfrak{A}, C_{0}\right)(L(\mathbf{a a}))\left((\mathfrak{B}, D) \vDash \varphi[D]\right.$ iff $(\mathfrak{B}, D) \equiv_{n-1}\left(\mathfrak{A}, C_{0}\right)(L($ aa $))$ respectively).

We omit the proof of this well-known fact (see also [10, pp. 58-59]). Moreover we can assume that $\operatorname{qr}(\varphi(X)) \leqslant n$. By (ii) and the choice of $C_{0}$ we get $\mathfrak{A} F$ $(\operatorname{aa} X) \varphi(X)$. Hence by (i) $\mathfrak{B} \vDash(\operatorname{aa} X) \varphi(X)$. By the definition of $\varphi(X)$ we get the existence of a cub system $\mathscr{D}$ from $B$ such that $(\forall C \in \mathcal{C})(\forall D \in \mathscr{D})(\mathfrak{A}, C)$ $\equiv_{n-1}(\mathfrak{B}, D)(L($ aa $))$. Q.E.D.

The following well-known result concludes this section.

Lemma 1.9. Let $A$ be a set of cardinality $\omega_{1}$. Then there is a cub collection $\mathbb{Q}$ on $A$ which is well-ordered of type $\omega_{1}$ by inclusion.

2. Ordered sums of finitely determinate linear orderings. Key result of this article is the following theorem.

THEOREM 2.1. Let $L_{1}, L_{2}$ be extensions of the language $L$ for linear orderings by $a$ finite number of relation symbols. Moreover let $n$ be a natural number and let $\mathfrak{A}, \mathfrak{B}$ be finitely $n$-determinate structures for $L_{1}$. For each $a \in A, b \in B$ let $\mathfrak{C}_{a}, \mathfrak{D}_{b}$ be finitely $n$-determinate structures for $L_{2}$. Let $\mathfrak{A} \equiv_{n} \mathfrak{B}\left(L_{1}(\mathbf{a a})\right), \mathfrak{E}_{a} \equiv_{n} \mathfrak{D}_{b}\left(L_{2}(\mathbf{a a})\right)$ (for all $a \in A, b \in B)$ be fulfilled, and let $L_{3}=L_{1} \cup L_{2}$. If $\mathfrak{A}, \mathfrak{B}$ and all $\mathfrak{E}_{a}, \mathfrak{D}_{b}(a \in A$, $b \in B)$ are linear orderings, then

$$
\sum_{\mathfrak{A}} \mathfrak{E}_{a} \equiv_{n} \sum_{\mathfrak{B}} \mathfrak{D}_{b}\left(L_{3}(\mathbf{a a})\right)
$$

holds and both structures are finitely $n$-determinate. 
REMARK 2.2. This theorem is a generalization (done by the referee) of a corresponding result in the first version of this article. There only structures of cardinality $\leqslant \omega_{1}$ were regarded and $L_{1}, L_{2}$ were extensions of $L$ by a finite number of unary relation symbols. The following proof we take from the referee's report.

We need the following

Lemma 2.3. Suppose $\mathfrak{A}$ and $\mathfrak{B}$ are any structures for the same finite language $L^{\prime}$, and suppose $f \subseteq A * B, k<l<\omega, a \in A$, and $(\mathfrak{A}, \operatorname{dom} f) \equiv_{l}(\mathfrak{B}, \operatorname{rn} f)\left(L^{\prime}(\mathbf{a a})\right)$. Then for some $b \in B,(\mathfrak{A}, \operatorname{dom} f, a) \equiv_{k}(\mathfrak{B}, \operatorname{rn} f, b)(\mathbf{a a})$.

Proof. Clear, since there are only finitely many formulas of quantifier rank at most $k$ (up to equivalence) in a fixed finite number of variables. Q.E.D.

Proof of Theorem 2.1. At first we assume that all structures $\mathbb{E}_{a}$, $\mathfrak{D}_{b}$ (for $a \in A, b \in B)$ are uncountable. Let us write $\mathfrak{E}=\Sigma_{\mathfrak{A}} \mathfrak{E}_{a}, \mathfrak{D}=\Sigma_{\mathfrak{g}} \mathfrak{D}_{b}$; we may assume $C \cap D=\varnothing$. Here is some further notation:

For $x \in C$, write $x^{*}=a$ iff $x \in C_{a}$.

For $y \in D$, write $y^{*}=b$ iff $y \in D_{b}$.

For $s \in P_{\omega_{1}}(C)$, write $s^{*}=\left\{x^{*}: x \in S\right\}$; thus, $s^{*} \in P_{\omega_{1}}(A)$.

For $t \in P_{\omega_{1}}(D)$, write $t^{*}=\left\{y^{*}: y \in t\right\}$; thus, $t^{*} \in P_{\omega_{1}}(B)$.

For $f \subseteq C * D, a \in A$, and $b \in B$, define $\operatorname{dom}_{a} f$ to be the trace of $f$ on $C_{a}$. More precisely:

for $a \in A, \operatorname{dom}_{a} f:=\left\{x: x^{*}=a \& x \in \operatorname{dom} f\right\} \cup\left\{s \cap C_{a}: s \cap C_{a} \neq \varnothing \&\right.$ $s \in \operatorname{dom} f\}$,

for $b \in B, \operatorname{rn}_{b} f:=\left\{y: y^{*}=b \& y \in \operatorname{rn} f\right\} \cup\left\{t \cap D_{b}: t \cap D_{b} \neq \varnothing \& t \in\right.$ rn $f\}$ and

$f^{*}:=\left\{\left(u^{*}, v^{*}\right):(u, v) \in f\right\}$. So if $f^{*}$ is a function, then $f^{*}\left(u^{*}\right)=(f(u))^{*}$.

For $a \in A$ and $b \in B$,

$$
\begin{aligned}
f_{a b}:= & {\left[f \cap\left(C_{a} \times D_{b}\right)\right] } \\
& \cup\left\{\left(s \cap C_{a}, t \cap D_{b}\right):(s, t) \in f \& s \cap C_{a} \neq \varnothing \& t \cap D_{b} \neq \varnothing\right\} .
\end{aligned}
$$

Notice that $\operatorname{dom} f_{a b} \subseteq \operatorname{dom}_{a} f$ and $\mathrm{rn} f_{a b} \subseteq \mathrm{rn}_{b} f$. Let $\left(G_{i}: i \leqslant n\right)$ be a determinate $\left(L_{1}(\mathbf{a a}), n\right)$ back-and-forth system from $\mathfrak{U}$ to $\mathfrak{B}$ (by Theorem 1.7).

Now define $\left(F_{i}: i \leqslant n\right)$ as follows: $f \in F_{i}$ iff

(I) $f$ is a finite function, and $f \subseteq A * B$.

(II) $f^{*} \in G_{i}$; in particular, $f^{*}$ is a function.

(III) $(\forall a \in A)(\forall b \in B)\left(\mathbb{E}_{a}, \operatorname{dom} f_{a b}\right) \equiv_{i}\left(\mathfrak{D}_{b}, \mathrm{rn} f_{a b}\right)(\mathbf{a a})$.

(IV) $(\forall a \in A)(\forall b \in B)\left(\mathfrak{E}_{a}, \operatorname{dom}_{a} f\right)$ and $\left(\mathfrak{D}_{b}, \mathrm{rn}_{b} f\right)$ are finitely $i$-determinate.

(V) (Here is a technical requirement to guarantee that each $f_{a b}$ is a function.) Suppose $s, t \in \operatorname{dom} f \cap P_{\omega_{1}}(C), s \neq t, a \in A, s \cap C_{a} \neq \varnothing$, and $t \cap C_{a} \neq \varnothing$. Then $s \cap C_{a} \neq t \cap C_{a}$.

We now check the conditions (1), (2) and (3) from Definition 1.6. (2) is immediate, i.e. $\varnothing \in F_{n}$, by hypothesis (and since $\varnothing \in G_{n}$, to verify (II) above).

For (1), we have to check that

$$
\mathfrak{C} \vDash \varphi(\operatorname{dom} f) \text { iff } \mathfrak{D} \vDash \varphi(\mathrm{rn} f)
$$

for all $f \in F_{0}$. By symmetry, we check only the $\Rightarrow$ direction. Let $f \in F_{j}$. 
Case 1. $\varphi(\operatorname{dom} f)$ is $p<q$. If $p^{*}=q^{*}$, we are done by (III) since $f \in F_{0}$, taking $a=p^{*}, b=(f(p))^{*}$.

Otherwise, without loss of generality $p^{*}<q^{*}$; then $f^{*}\left(p^{*}\right)<f^{*}\left(q^{*}\right)$ by (II); i.e. $(f(p))^{*}<(f(q))^{*}$ by definition of $f^{*}$; so $f(p)<f(q)$ by definition of ordered sum.

Case 2. $\varphi(\operatorname{dom} f)$ is $p \in s$. Suppose $p \in s$; we show $f(p) \in f(s)$. Let $a=p^{*}$, $b=f^{*}(a)$. Then $\left(\mathcal{E}_{a}, \operatorname{dom} f_{a b}\right) \vDash p \in\left(s \cap C_{a}\right)$. Now since $p \in s \cap C_{a}$ and $f(p) \in$ $f(s) \cap D_{b}, s \cap C_{a} \neq \varnothing$ and $f(s) \cap D_{b} \neq \varnothing$. So by definition of $f_{a b}$ (which is a function by $(\mathrm{V}))$,

$$
f_{a b}\left(s \cap C_{a}\right)=f(s) \cap D_{b} .
$$

Hence $\left(\mathfrak{D}_{b}, \operatorname{rn} f_{a b}\right) \vDash f(p) \in\left(f(s) \cap D_{b}\right)$, by (III). Therefore $f(p) \in f(s)$, as desired.

Case 3. $\varphi(\operatorname{dom} f)$ is $R\left(p_{1}, \ldots, p_{n}\right)$, for $R$ from $L_{2}$. But if $R_{\mathbb{S}}\left(p_{1}, \ldots, p_{n}\right)$, then $p_{1}^{*}=\cdots=p_{n}^{*}$ by definition of ordered sum, so $R_{\mathscr{D}}\left(f\left(p_{1}\right), \ldots, f\left(p_{n}\right)\right)$ holds by (III).

Case 4. $\varphi\left(\right.$ dom f) is $R\left(p_{1}, \ldots, p_{n}\right)$, for $R$ from $L_{1}$. If $R_{\mathscr{E}}\left(p_{1}, \ldots, p_{n}\right)$, then $R_{\mathscr{U}}\left(p_{1}^{*}, \ldots, p_{n}^{*}\right)$ by definition of ordered sum. By (II) we get $R_{\mathfrak{g}}\left(f^{*}\left(p_{1}^{*}\right), \ldots, f^{*}\left(p_{n}^{*}\right)\right)$. Hence $R_{\mathfrak{g}}\left(\left(f\left(p_{1}\right)\right)^{*}, \ldots,\left(f\left(p_{n}\right)\right)^{*}\right)$. This gives $R_{\mathfrak{D}}\left(f\left(p_{1}\right), \ldots, f\left(p_{n}\right)\right)$ by definition of ordered sum.

Now we check (3)(i) from Definition 1.6; (3)(ii) is similar. Suppose $f \in F_{l}, k<l$, and $c \in C$, say $c \in C_{a_{0}}$; we find $d$ such that $f \cup\{(c, d)\} \in F_{k}$. First choose $b_{0}$ such that $f^{*} \cup\left\{\left(a_{0}, b_{0}\right)\right\} \in G_{k}$, by (II). Then choose $d \in D_{b_{0}}$ such that

$$
\left(\mathfrak{S}_{a_{0}}, \operatorname{dom} f_{a_{0} b_{0}}, c\right) \equiv_{k}\left(\mathfrak{D}_{b_{0}}, \mathrm{rn} f_{a_{0} b_{0}}, d\right)(\mathbf{a a}) \text {; }
$$

this can be done by (III) for $F_{l}$, together with Lemma 2.3. Set $f^{\prime}=f \cup\{(c, d)\}$. Conditions (I) and (V) clearly remain true for $f^{\prime}$. (II) is true for $f^{\prime} \in F_{k}$ by choice of $b$. (III) trivially remains true for $f^{\prime}$ for all $a, b$ with $a \neq a_{0}$ or $b \neq b_{0}$, since then $f_{a_{0}}=f_{a_{0} b_{0}}$. For $a=a_{0}$ and $b=b_{0}$ (III) is true for $f^{\prime}$ by choice of $d$. (IV) remains true for $f^{\prime} \in F_{k}$ because $l$-determinancy obviously yields $(l-1)$-determinancy when a new constant is named, and $l-1 \geqslant k$.

Finally, we check (3)(iii). Given $k<l<\omega$ and $f \in F_{l}$, pick for each $a \in A$ and $b \in B$ cub sets $\mathscr{X}_{a} \subseteq P_{\omega_{1}}\left(C_{a}\right), \mathscr{Y}_{b} \subseteq P_{\omega_{1}}\left(D_{b}\right)$ such that

$$
\begin{aligned}
& \forall s_{0} \in \mathcal{X}_{a}, \quad\left(\mathfrak{S}_{a}, \operatorname{dom}_{a} f\right) \vDash\left[(\text { aas }) \varphi(s) \rightarrow \varphi\left(s_{0}\right)\right] ; \\
& \forall t_{0} \in \mathcal{Y}_{b}, \quad\left(\mathfrak{D}_{b}, \operatorname{rn}_{b} f\right) \vDash\left[(\operatorname{aas}) \varphi(s) \rightarrow \varphi\left(t_{0}\right)\right] .
\end{aligned}
$$

To do this for any particular $\varphi$ is possible by the definition of satisfaction in $L$ (aa); then by countable completeness, we can handle all $\varphi$ at once. (In fact, the proof will show that it suffices to consider only $\varphi$ of quantifier rank $\leqslant k$; so finite completeness suffices.) We want somehow to glue the different $\mathcal{X}_{a}$ together and the different $\mathscr{Y}_{b}$ together to verify (3)(iii). To this end, choose cub sets $\mathcal{E}_{A}^{*} \subseteq P_{\omega_{1}}(A)$, $\mathcal{E}_{B}^{*} \subseteq P_{\omega_{1}}(B)$ such that

$$
\left(\forall s \in \mathcal{E}_{A}^{*}\right)\left(\forall t \in \mathcal{E}_{B}^{*}\right)\left[f^{*} \cup\{(s, t)\} \in G_{k}\right] .
$$

Here we are using condition (II) for $f \in F_{l}$, together with (3)(iii) (from Definition 1.6) for $f^{*} \in G_{l}$. Now define cub sets 


$$
\begin{aligned}
& \mathcal{E}_{A}:=\left\{s: s \in P_{\omega_{1}}(C) \& s^{*} \in \mathcal{E}_{A}^{*}\right\}, \\
& \mathcal{E}_{B}:=\left\{s: s \in P_{\omega_{1}}(D) \& s^{*} \in \mathcal{E}_{B}^{*}\right\} .
\end{aligned}
$$

The following sets are clearly cub:

$$
\begin{aligned}
& \mathscr{Z}_{A}:=\left\{s: s \in P_{\omega_{1}}(C) \& \forall a \in A, s \cap C_{a} \in \mathcal{X}_{a} \text { or } s \cap C_{a}=\varnothing\right\} ; \\
& \mathscr{Z}_{B}:=\left\{s: s \in P_{\omega_{1}}(D) \& \forall b \in B, s \cap D_{b} \in \mathscr{Y}_{b} \text { or } s \cap D_{b}=\varnothing\right\} .
\end{aligned}
$$

Finally, set

$$
\mathcal{H}_{C}:=\mathcal{E}_{A} \cap \mathscr{Z}_{A} \cap\left\{s: s \supsetneqq \bigcup\left[(\operatorname{dom} f) \cap P_{\omega_{1}}(C)\right]\right\}, \quad \mathcal{H}_{D}:=\mathcal{E}_{B} \cap \mathscr{Z}_{B} .
$$

Clearly $\mathcal{H}_{C}$ and $\mathcal{H}_{D}$ are cub, being finite intersections of cub sets. The proof is thus completed when we prove the following

Claim. $\left(\forall s \in \mathcal{H}_{C}\right)\left(\forall t \in \mathcal{K}_{D}\right)\left[f \cup\{(s, t)\} \in F_{k}\right]$.

For, choose any $s_{0} \in \mathcal{H}_{C}, t_{0} \in \mathcal{H}_{D}$. We check (I) through (V) in the definition of $F_{k}$. (I) is obvious. (II) holds by (*). For (III), choose $a \in A$ and $b \in B$. Note first that if $s_{0} \cap C_{a}=\varnothing$ or $t_{0} \cap D_{b}=\varnothing$, then (by definition of $\left.f_{a b}\right)\left(f \cup\left\{\left(s_{0}, t_{0}\right)\right\}\right)_{a b}=$ $f_{a b}$, and therefore (III) holds since $f \in F_{l}$. Otherwise $s_{0} \cap C_{a} \neq \varnothing$ and $t_{0} \cap D_{b} \neq$ $\varnothing$, so since $s_{0} \in \mathcal{H}_{C} \subseteq \mathscr{Z}_{A}$ and $t_{0} \in \mathcal{H}_{D} \subseteq \mathscr{Z}_{B}$,

$$
s_{0} \cap C_{a} \in \mathscr{X}_{a}
$$

and

$$
t_{0} \cap D_{b} \in \mathscr{Y}_{b},
$$

by definition of $\mathscr{Z}_{A}$ and $\mathscr{Z}_{B}$. Now recalling that $\operatorname{dom} f_{a b} \subseteq \operatorname{dom}_{a} f$ and $\operatorname{rn} f_{a b} \subseteq$ $\mathrm{rn}_{b} f$, and assuming $\operatorname{qr}(\varphi) \leqslant k$, we have the following implications:

$$
\begin{aligned}
& \left(\mathfrak{\Im}_{a}, \operatorname{dom} f_{a b}\right) \vDash \varphi\left(\operatorname{dom} f_{a b}, s_{0} \cap C_{a}\right) \\
& \Rightarrow\left(\mathfrak{(}_{a}, \operatorname{dom} f_{a b}\right) \vDash \neg(\text { aas }) \neg \varphi\left(\operatorname{dom} f_{a b}, s\right)
\end{aligned}
$$

(by the contra-positive of (1) above, and by (3) above)

$$
\Rightarrow\left(\mathfrak{夭}_{a}, \operatorname{dom} f_{a b}\right) \vDash(\operatorname{aas}) \varphi\left(\operatorname{dom} f_{a b}, s\right)
$$

(by $l$-determinancy of $\left(\mho_{a}, \operatorname{dom} f_{a b}\right)$, using (IV))

$$
\begin{aligned}
& \left.\Rightarrow\left(\mathfrak{D}_{b}, \mathrm{rn} f_{a b}\right) \vDash(\operatorname{aas}) \varphi\left(\operatorname{rn} f_{a b}, s\right) \quad \text { (by (III) for } f \in F_{l}\right) \\
& \Rightarrow\left(\mathfrak{D}_{b}, \operatorname{rn} f_{a b}\right) \vDash \varphi\left(\operatorname{rn} f_{a b}, t_{0} \cap D_{b}\right) \quad \text { (by (2) and (4) above). }
\end{aligned}
$$

Similarly, if $\left(\mathfrak{D}_{b}, \mathrm{rn} f_{a b}\right) \vDash \varphi\left(\mathrm{rn} f_{a b}, t_{0} \cap D_{b}\right)$ then $\left(\mathbb{E}_{a}, \operatorname{dom} f_{a b}\right) \vDash \varphi\left(\operatorname{dom} f_{a b}, s_{0}\right)$.

So

$$
\left(\mathfrak{S}_{a}, \operatorname{dom}\left(f \cup\left\{\left(s_{0}, t_{0}\right)\right\}\right)_{a b}\right) \equiv_{k}\left(\mathfrak{D}_{b}, \operatorname{rn}\left(f \cup\left\{\left(s_{0}, t_{0}\right)\right\}\right)_{a b}\right)(\mathbf{a a})
$$

verifying (III).

To check (IV), fix $a \in A$. If $s_{0} \cap C_{a}=\varnothing$, then $\left[f \cup\left\{\left(s_{0}, t_{0}\right)\right\}\right]_{a}=f_{a}$, so we are done by (IV) for $f \in F_{l}$ : so assume $s_{0} \cap C_{a} \neq \varnothing$. Suppose $\operatorname{qr}(\varphi) \leqslant k$, where $\varphi(s)$ is

$$
(\mathbf{a a t} \vec{t})(\forall \vec{x})[(\text { stat } Y) \psi(s, \vec{t}, Y, \vec{x}) \rightarrow(\operatorname{aa} Y) \psi(s, \vec{t}, Y, \vec{x})] \text {, }
$$

and $\psi$ may have parameters from $\operatorname{dom}_{a} f$. We must show

$$
\left(\mathcal{E}_{a}, \operatorname{dom}_{a}\left(f \cup\left\{\left(s_{0}, t_{0}\right)\right\}\right)\right) \vDash \varphi\left(s_{0} \cap C_{a}\right) \text {. }
$$


By hypothesis,

$$
\left(\Subset_{a}, \operatorname{dom}_{a} f\right) \vDash(\text { aas }) \varphi(s) .
$$

Since we have assumed $s_{0} \cap C_{a} \neq \varnothing$, then by definition of $\mathscr{Z}_{A} s_{0} \cap C_{a} \in \mathcal{X}_{a}$; hence

$$
\left(\varsigma_{a}, \operatorname{dom}_{a}\left(f \cup\left\{\left(s_{0}, t_{0}\right)\right\}\right)\right) \vDash \varphi\left(s_{0} \cap C_{a}\right)
$$

by (1) and (5) above. So $\left(\mathbb{E}_{a}\right.$, $\left.\operatorname{dom}_{a}\left(f \cup\left\{\left(s_{0}, t_{0}\right)\right\}\right)\right)$ is finitely $k$-determinate. Similarly, $\left(\mathfrak{D}_{b}, \mathrm{rn}_{b}\left(f \cup\left\{\left(s_{0}, t_{0}\right)\right\}\right)\right)$ is finitely $k$-determinate.

Observe that $(\mathrm{V})$ is guaranteed because

$$
s_{0} \in \mathcal{K}_{C} \subseteq\left\{s: s \supsetneq \bigcup\left[(\operatorname{dom} f) \cap P_{\omega_{1}}(C)\right]\right\} .
$$

Now we regard the case that all stuctures $\mathfrak{E}_{a}, \mathfrak{D}_{b}$ (for $a \in A, b \in B$ ) are of cardinality $<\omega_{1}$.

In this case the definition of $\left(F_{i}: i \leqslant n\right)$ is to change. As above take conditions (I), (II) and (III) and substitute the conditions (IV) and (V) by the following condition:

(VI) $\left(\operatorname{dom}_{a} f\right) \cap P_{\omega_{1}}\left(C_{a}\right)=C_{a}$ for all $a \in A$ with $\left(\operatorname{dom}_{a} f\right) \cap P_{\omega_{1}}\left(C_{a}\right) \neq \varnothing$ and $\left(\mathrm{rn}_{b} f\right) \cap P_{\omega_{1}}\left(D_{b}\right)=D_{b}$ for all $b \in B$ with $\left(\mathrm{rn}_{b} f\right) \cap P_{\omega_{1}}\left(D_{b}\right) \neq \varnothing$.

The old condition (IV) is obviously fulfilled since $C_{a}$ and $D_{b}$ are of cardinality < $\omega_{1}$. We can use $\left\{C_{a}\right\}$ and $\left\{D_{b}\right\}$ as corresponding cub sets. The new condition (VI) guarantees instead of condition (V) that each $f_{a b}$ is a function.

Using these remarks we can take the same proof as above to check conditions (1), (2) and (3) from Definition 1.6.

It remains to regard the case where some of the stuctures $\mathfrak{E}_{a}, \mathfrak{D}_{b}$ (for $a \in A$, $b \in B)$ are uncountable and some are not. But this happens only if $n \leqslant 1$. However in this case the theorem is trivial. Q.E.D.

3. Finitely determinate linear orderings and decidability. The following corollaries are simple consequences of Theorem 2.1.

COROLlaRy 3.1. Let $\mathfrak{A}, \mathfrak{B}, \mathfrak{C}$ and $\mathfrak{D}$ be finitely $n$-determinate linear orderings such that $\mathfrak{A} \equiv_{n} \mathfrak{B}(L(\mathbf{a a}))$ and $\mathfrak{E} \equiv_{n} \mathfrak{D}(L(\mathbf{a a}))$ are fulfilled. Then $\mathfrak{A}+\mathbb{E}$ and $\mathfrak{A} \cdot \mathbb{E}$ are finitely $n$-determinate and $\mathfrak{A}+\mathfrak{E} \equiv_{n} \mathfrak{B}+\mathfrak{D}(L(\mathbf{a a}))$ and $\mathfrak{A} \cdot \mathfrak{E} \equiv_{n} \mathfrak{B} \cdot \mathfrak{D}(L(\mathbf{a a}))$ hold.

COROLlaRy 3.2. Let $\mathfrak{A}$ be a linear ordering of cofinality $\omega$ which has a first element and the property that each interval of $\mathfrak{A}$ is finitely $n$-determinate. Then also $\mathfrak{A}$ is finitely $n$-determinate.

Corollary 3.1 follows immediately from Theorem 2.1.

Proof of Corollary 3.2. Let $\left(a_{n}\right)_{n \in \omega}$ be a cofinal sequence of elements of $A$ such that $a_{0}$ is the minimal element of $\mathfrak{A}$. For all $a, b \in A$ let $[a, b)$ be the structure

$$
\text { ( } \left.\{c: a \leqslant c<b \& c \in A\},<_{\mathscr{A}} \uparrow\{c: a \leqslant c<b \& c \in A\}\right) \text {. }
$$

For each given $n \in \omega$ there are only finitely many equivalence classes with respect to $\equiv_{n}(L(\mathbf{a a}))$. A standard application of the theorem of Ramsey [20] (see e.g. [12], [26]) gives then the existence of an infinite subset $N$ of $\omega$ such that for all 
$m_{1}, m_{2}, m_{3}, m_{4} \in N$ with $m_{1}<m_{2}$ and $m_{3}<m_{4},\left[a_{m_{1}}, a_{m_{2}}\right) \equiv_{n}\left[a_{m_{3}}, a_{m_{4}}\right)($ aa) holds. Let $\left(n_{i}\right)_{i \in \omega}$ be an enumeration of the elements of $N$ with $n_{i}<n_{j}$ for all $i<j \in \omega$. We get

$$
A=\left[a_{0}, a_{n_{0}}\right)+\sum_{i \in \omega}\left[a_{n_{i}}, a_{n_{i+1}}\right)
$$

But the intervals $\left[a_{0}, a_{n_{0}}\right)$ and $\left[a_{n_{i}}, a_{n_{i+1}}\right)$ (for $i \in \omega$ ) are finitely $n$-determinate. Hence by Theorem 2.1 and Corollary $3.1\left[a_{0}, a_{n_{0}}\right)+\sum_{i \in \omega}\left[a_{n_{i}}, a_{n_{i+1}}\right)$ is finitely n-determinate. Q.E.D.

The next theorem solves the second part of Question 7.2 of [10] (see also [7]).

THEOREM 3.3. All ordinals are finitely determinate.

REMARK 3.4. The proof of this result in a first version of this article contained a mistake. The proof was corrected by the referee of this article. The following proof was done by the referee. At the same time Alan Mekler [16] (see also [17]) found a different proof of this result.

Proof. Let $n \in \omega$. We show that each ordinal is finitely $n$-determinate. If not, then there is a minimal ordinal $\alpha$ which is not finitely $n$-determinate. By Corollary 3.1 and Corollary 3.2, $c f(\alpha)>\omega$ and $\alpha$ is closed under addition. It follows that the following set $X$ is unbounded in $\alpha$ :

$$
X:=\{\beta: \beta<\alpha \& \beta \text { is closed under }+\} .
$$

Since there are only finitely many sentences of the language for linear orderings of quantifier rank $\leqslant n$ (up to equivalence), there is some set $Y \subseteq X$ such that $Y$ is unbounded and

$$
\text { for all } \beta, \gamma \in Y, \quad(\beta,<) \equiv_{n}(\gamma,<)(L(\mathbf{a a})) .
$$

Choose a sentence $\varphi$ of quantifier rank $n$ such that for all $(A,<),(A,<) \vDash \varphi$ iff $(A,<) \equiv_{n}(\beta,<)(L(\mathbf{a a}))$ for all (or any) $\beta \in Y$.

Now let $\sqrt{E}=\left(C,<_{\mathfrak{E}},+_{\mathfrak{E}}\right)$ be a model of size $\omega_{1}$ such that

$$
\Subset \equiv(\alpha,<,+)(L(\mathbf{a a})) \text {, }
$$

by the downward Löwenheim-Skolem Theorem for $L$ (aa) (see [1]). For $c, d \in C$, write $\left(c,<_{)}\right)$for $\left(\left\{x: x<_{\mathbb{E}} c\right\},<_{c} \uparrow\left\{x: x<_{\mathbb{G}} c\right\}\right)$, and write $[d, c)$ for $(\{x: d=x$ or

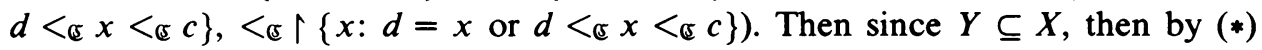
and the definition of $\varphi$, the following set $S$ is unbounded in $\Subset$ :

$$
S:=\left\{c: c \in C \&(c,<) \text { is closed under }+_{\sigma} \&(c,<) \vDash \varphi\right\} .
$$

Notice that for all $c \in S$ and $d<c,+_{\mathbb{C}}$ gives an isomorphism

$$
(c,<) \cong[d, c) \text {. }
$$

Now notice that $(\operatorname{aas})(\exists x)(x=\sup s)$ holds in $\mathbb{E}$, since it holds in $(\alpha,<)$. So there is a strictly increasing sequence $\left(c_{\alpha}: \alpha<\omega_{1}\right)$ from $C$ such that $c_{\lambda}=\sup _{\alpha<\lambda} c_{\alpha}$ for limit $\lambda$. Now choose a strictly increasing, unbounded sequence $\left(s_{\alpha}: \alpha<\omega_{1}\right)$ from $S$, such that for all $\alpha<\omega_{1}, s_{\alpha}>c_{\beta}$ for some $\beta$, such that $c_{\beta}>s_{\gamma}$ for all $\gamma<\alpha$. Write $s_{\beta}^{*}$ for $\sup _{\alpha<\beta} s_{\alpha}$; thus $s_{\beta}^{*}=s_{\alpha}$ if $\beta=\alpha+1$, and $s_{\beta}^{*}=c_{\gamma}$ for some $\gamma$ if $\beta$ is a limit. 
Then

$$
(C,<) \cong \sum_{\left(\omega_{1},<\right)}\left[s_{\alpha}^{*}, s_{\alpha}\right) \cong \sum_{\left(\omega_{1},<\right)}\left(s_{\alpha},<\right),
$$

the last $\cong$ by $(* *)$. But for all $\alpha, \beta<\omega_{1},\left(s_{\alpha},<\right) \equiv_{n}\left(s_{\beta},<\right)(L($ aa) $)$ by definition of $S$. Also, since $\left(C,<_{\S}\right) \equiv(\alpha,<)(L(\mathbf{a a}))$, each $\left(s_{\alpha},<\right)$ is finitely determinate. Therefore Theorem 2.1 yields that $\left(C,<_{\mathfrak{G}}\right)$ is finitely $n$-determinate, as desired. Q.E.D.

THEOREM 3.5. Let $O N$ be the class of all ordinals and let $K$ be the smallest (under inclusion) class of ordinals which contains $1, \omega$ and $\omega_{1}$, and is closed by the operations + and $\cdot\left(\right.$ if $\alpha, \beta \in K$, then also $\alpha+\beta, \alpha \cdot \beta \in K$ ). Then $\operatorname{Th}_{\mathrm{aa}}(K)=$ $\mathrm{Th}_{\mathrm{aa}}(O N)$.

Proof. It is sufficient to prove that for each sentence $\varphi$ of $L(\mathbf{a a})$ which has a model in the class $O N$ there is an $\alpha \in K$ with $\alpha \vDash \varphi$. Assume not, then there is an ordinal $\beta$ and a sentence $\varphi$ of $L$ (aa) with $K \vDash \neg \varphi$ and $\beta \vDash \varphi$. Now let $n=\operatorname{qr}(\varphi)$ and let $\beta^{\prime}$ be the smallest ordinal with the property that there does not exist an $\alpha \in K$ with $\alpha \equiv_{n} \beta^{\prime}(L(\mathbf{a a}))$. Hence for all $\beta^{\prime \prime}<\beta^{\prime}$ there is an $\alpha \in K$ with $\alpha$ $\equiv_{n} \beta^{\prime \prime}(L(\mathbf{a a}))$.

In [7], Theorem 2.6 is proved that each finitely determinate structure has an $L\left(\right.$ aa)-elementary substructure of cardinality $\leqslant \omega_{1}$. Hence $\beta^{\prime}<\omega_{2}$, by Theorem 3.3. By Corollary 3.1, $\beta^{\prime}$ is closed under addition. $c f\left(\beta^{\prime}\right)>\omega$ can be proved similarly to Corollary 3.2. We have to remember here that for each $\gamma<\delta<\beta^{\prime}$ there exists a $\tau \in K$ with

$$
(\{\nu: \gamma \leqslant \nu<\delta\},<\uparrow\{\nu: \gamma \leqslant \nu<\delta\}) \equiv_{n} \tau(L(\text { aa })) .
$$

Now let $\left(\beta_{\nu}\right)_{\nu<\omega_{1}}$ be a sequence which is cofinal in $\beta^{\prime}$. Moreover we can assume $\left(\beta_{\nu},<\right) \equiv_{n}(\beta \mu,<)\left(L(\right.$ aa) $)$ (for all $\left.\nu, \mu \in \omega_{1}\right)$, since $L$ is a finite language. Then $\beta^{\prime}=\sum_{\omega_{1}} \beta_{\nu}$, since $\beta^{\prime}$ is closed under addition and $c f\left(\beta^{\prime}\right)>\omega$. But since $\beta_{0}<\beta^{\prime}$ there is an $\alpha \in K$ with $\beta_{0} \equiv_{n} \alpha(L($ aa $))$. Hence $\beta^{\prime} \equiv_{n} \alpha \cdot \omega_{1}(L($ aa $))$, by Theorem 2.1. Q.E.D.

THEOREM 3.6. Let $L_{1}, L_{2}$ be extensions of the language $L$ for linear orderings by $a$ finite number of relation symbols. Let $\mathfrak{A}, \mathfrak{B}$ be finitely determinate linear orderings for $L_{1}, L_{2}$ respectively. Moreover let $\mathrm{Th}_{L_{1}(\mathrm{a})(}(\mathfrak{A})$ and $\mathrm{Th}_{L_{2}(\mathrm{~m})(}(\mathfrak{B})$ be axiomatizable, and let $L_{3}=L_{1} \cup L_{2}$. Then $\mathrm{Th}_{L_{3}(\mathfrak{a})}(\mathfrak{U}+\mathfrak{B})$ and $\mathrm{Th}_{L_{3}(\mathfrak{a})}(\mathfrak{U} \cdot \mathfrak{B})$ are axiomatizable.

The proof of this theorem is simple, but lengthy. Hence we shall give only some hints and leave the details to the reader. At first show that $1+\mathfrak{B}$ and $1+\mathfrak{A}+1$ are axiomatizable. Then extend the corresponding languages by new unary predicates which define the minimal (the minimal and the maximal) element of $1+\mathfrak{B}$ $(1+\mathfrak{U}+1$ respectively). Let $\mathfrak{C}, \mathfrak{D}$ be the corresponding extended structures. Then it is easy to prove that $\operatorname{Th}_{\mathfrak{a g}}(\mathfrak{H}+\mathfrak{E})$ and $\mathrm{Th}_{\mathfrak{a q}}(\mathfrak{D} \cdot \mathfrak{B})$ are axiomatizable (use Theorem 2.1). But this gives the axiomatizability of $\mathfrak{A}+\mathfrak{B}$ and $\mathfrak{A} \cdot \mathfrak{B}$, since these structures are definable in $\mathfrak{A}+\mathfrak{E}, \mathfrak{D} \cdot \mathfrak{B}$ respectively.

The structures $\left(\omega_{1}^{n},<\right)$ (for $0<n \in \omega$ ) and $R$ (ordering of real numbers) are finitely determinate and have a decidable theory in stationary logic (for a proof see [7]). Obviously $\omega_{1}^{*}$ (the inverse ordering of $\omega_{1}$ ) is also finitely determinate and has a 
decidable theory in stationary logic. Together with Theorem 3.6 and Corollary 3.1 this gives the following result.

THEOREM 3.7. Let $D$ be the smallest (under inclusion) class of linear orderings which contains $1, \omega, \omega_{1}, \omega^{*}, \omega_{1}^{*}, \eta$ (ordering of rational numbers) and $R$ and is closed under the operations + and $\cdot$. Then for each $\alpha \in D, \operatorname{Th}_{\alpha a}(\alpha)$ is axiomatizable and hence decidable. The axiomatization can be found effectively depending on $\alpha$.

TheOREM 3.8. Let $O N$ and $K$ be as in Theorem 3.5 and let $D$ be as in Theorem 3.7. Then $\mathrm{Th}_{\mathrm{aa}}(D), \mathrm{Th}_{\mathrm{ag}}(K)$ and hence $\mathrm{Th}_{\mathrm{aa}}(O N)$ are decidable.

Proof. At first we define for each $\alpha \in D$ the depth of $\alpha$, denoted by $d(\alpha)$.

$$
\begin{gathered}
d(1):=d(\omega):=d\left(\omega_{1}\right):=d\left(\omega^{*}\right):=d\left(\omega_{1}^{*}\right):=d(\eta):=d(R):=1, \\
d(\alpha+\beta):=\max (d(\alpha), d(\beta))+1, d(\alpha \cdot \beta):=\max (d(\alpha), d(\beta))+1 .
\end{gathered}
$$

Let $L$ be the elementary language for linear orderings. Obviously there exists a recursive upper bound $f(n)$ for the number of equivalence classes of $\equiv_{n}(L(\mathbf{a a}))$.

Using Corollary 3.1 it is easy to see that for each $\alpha \in D$ with $d(\alpha)>f(n)+1$ there is an $\alpha^{\prime} \in D$ with $d\left(\alpha^{\prime}\right)<d(\alpha)$ and $\alpha^{\prime} \equiv_{n} \alpha\left(L(\right.$ aa) $)$. Let $\Delta_{n}$ be the set $\{\alpha$ : $\alpha \in D \& d(\alpha) \leqslant f(n)+1\}$. We get

$$
\Delta_{n} \vDash \varphi \text { iff } D \vDash \varphi
$$

for all sentences $\varphi$ of $L\left(\right.$ aa) with $\operatorname{qr}(\varphi) \leqslant n . \Delta_{n}$ is finite and $n \rightarrow \Delta_{n}$ is effective. Hence $\operatorname{Th}_{\mathrm{aa}}(D)$ is decidable by Theorem 3.7. The decidability of $\mathrm{Th}_{\mathrm{aa}}(K)$ can be proved similarly. Q.E.D.

It is interesting to remember that $\operatorname{Th}_{Q_{1}}(K)=\operatorname{Th}_{Q_{1}}(O N)$, i.e. $K$ is also a dense system for the $Q_{1}$-theory of well-orderings. This is e.g. a consequence of the results from [27]. But the quantifier aa is not eliminable in $\operatorname{Th}_{2 a}(O N)$ by $Q_{1}$. To see this we regard the following formula $\varphi$ :

$$
(\mathbf{a a} X)(\forall x)(\exists y) y \in X \wedge x<y .
$$

Obviously we have, for each limit ordinal $\alpha, \alpha \vDash \varphi$ iff $c f(\alpha) \leqslant \omega$. However this is not possible to express by a formula from $L\left(Q_{1}\right)$. This can be proved regarding the ordinals $\omega_{1}^{\omega_{1}}$ and $\omega_{1}^{\omega}$. The cofinality of $\omega_{1}^{\omega_{1}}$ is $\omega_{1}$ and the cofinality of $\omega_{1}^{\omega}$ is $\omega$. But by an induction on $n$ we get that

$$
\omega_{1}^{\omega_{1}} \equiv_{n} \omega_{1}^{\omega}\left(L\left(Q_{1}\right)\right)
$$

holds. This can be proved using the game theoretical equivalent of $\equiv_{n}\left(L\left(Q_{1}\right)\right)$ (see [13], [28]). Here it is essential to remember the following facts.

(1) For each natural number $n$ there is a natural number $m$ such that for each ordinal $\alpha$ there exists an ordinal $\beta<\omega_{1}^{m}$ with $\alpha \equiv_{n} \beta\left(L\left(Q_{1}\right)\right)$.

(2) For all ordinals $\alpha<\omega_{1}^{\omega_{1}}$ and $\beta<\omega_{1}^{\omega}, \alpha+\omega_{1}^{\omega_{1}} \cong \omega_{1}^{\omega_{1}}$ and $\beta+\omega_{1}^{\omega} \cong \omega_{1}^{\omega}$ hold.

If we regard the construction of the elements of $K$ and remember that $\equiv_{n}\left(L\left(Q_{1}\right)\right)$ has only finitely many equivalence classes then fact (1) follows simply from $\mathrm{Th}_{Q_{1}}(K)=\mathrm{Th}_{Q_{1}}(O N)$ (see also [3]). Fact (2) is obvious.

We leave the details to the reader and conclude this article with the following problems.

Problem 1. Is the aa-theory of the class of all finitely determinate linear orderings or Boolean algebras decidable? 
Problem 2. Is the aa-theory of the class of all linear orderings or Boolean algebras in $Z F+A D+D C$ decidable?

Problem 3. Is each tree of height $<\omega_{1}$ finitely determinate? In connection with this problem it is interesting that Alan Mekler [18] found a linear ordering which is not finitely determinate, but which contains no copy of either $\omega_{1}$ or $\omega_{1}^{*}$.

\section{REFERENCES}

1. J. Barwise, M. Kaufmann and M. Makkai, Stationary logic, Ann. Math. Logic 13 (1978), 171-224.

2. A. Baudisch, The elementary theory of Abelian groups with m-chains of pure subgroups, Fund. Math. (to appear).

3. A. Baudisch, D. G. Seese, H. P. Tuschik and M. Weese, Decidability and generalized quantifiers, Akademie-Verlag, Berlin, 1980.

4. A. Baudisch and H. P. Tuschik, $L(\mathbf{a})=L\left(Q_{1}\right)$ for trees, unpublished manuscript, 1979.

5. S. Ben-David, On Shelah's compactness of cardinals, Israel J. Math. 31 (1978), 34-56.

6. X. Caicedo, A back-and-forth characterization of elementary equivalence in stationary logic, preprint, 1977.

7. P. C. Eklof and A. H. Mekler, Stationary logic of finitely determinate structures, preprint, 1979.

8. H. Herre, A remark to a paper of $D$. Seese, unpublished manuscript, 1979.

9. H. Herre and H. Wolter, Entscheidbarkeit der linearen Ordnung in $L_{Q_{1}}, Z$. Math. Logik Grundlagen Math. 23 (1977), 273-282.

10. M. Kaufmann, Some results in stationary logic, Ph.D. Thesis, Univ. of Wisconsin, 1978.

11. K. Kunen, Combinatorics, Handbook of Mathematical Logic, edited by J. Barwise, North-Holland, Amsterdam, 1977, pp. 371-401.

12. H. Läuchli and J. Leonhard, On the elementary theory of linear order, Fund. Math. 59 (1966), 109-116.

13. L. D. Lipner, Some aspects of generalized quantifiers, Doctoral Dissertation, Univ. of California, Berkeley, 1970.

14. J. A. Makowsky, Elementary equivalence and definability in stationary logic, preprint, 1977.

15._, Quantifying over countable sets positive us. stationary logic, Logic Colloquium '77, edited by A. Macintyre, L. Pacholski and J. Paris, North-Holland, Amsterdam, 1978, pp. 183-193.

16. A. Mekler, All ordinals are finitely determinate, notes, 1979.

17. __ Every ordinal is finitely determinate. II, notes, 1979.

18. Another linear order which is not finitely determinate, notes, 1979.

19. M. O. Rabin, A simple method for undecidability proofs and some applications, Logic, Methodology and Philosophy of Science. II, edited by Y. Bar-Hillel, North-Holland, Amsterdam, 1964, pp. 58-68.

20. F. D. Ramsey, On a problem of formal logic, Proc. London Math. Soc. 30 (1929), 338-384.

21. D. Seese, Decidability and generalized quantifiers, Logic Colloquium '77, edited by A. Macintyre, L. Pacholski and J. Paris, North-Holland, Amsterdam, 1978, pp. 229-237.

22. __ Stationäre Logik-Beschränkte Mengen-Entscheidbarkeit, Dissertation (B), Berlin, 1979.

23. D. G. Seese, H. P. Tuschik and M. Weese, Linear orders and Boolean algebras in stationary logic, notes, 1979.

24. D. G. Seese and M. Weese, Ehrenfeucht's game for stationary logic, unpublished notes, 1977.

25. S. Shelah, Generalized quantifiers and compact logic, Trans. Amer. Math. Soc. 204 (1975), 342-364.

26. , The monadic theory of order, Ann. of Math. 102 (1975), 379-419.

27. H. P. Tuschik, On the decidability of the theory of linear orderings in the language $L\left(Q_{1}\right)$, Lecture Notes in Math., vol. 619, Springer-Verlag, Berlin, Heidelberg, New York, 1977, pp. 291-304.

28. _ On the decidability of the theory of linear orderings with generalized quantifiers, Fund. Math. (to appear).

29. S. Ulam, Zur Masstheorie in der allgemeinen Mengenlehre, Fund. Math. 16 (1930), 140-150.

30. S. Vinner, A generalization of Ehrenfeucht's game and some applications, Israel J. Math. 12 (1972), 279-298.

Zentralinstitut für Mathematik und Mechanik, ACademie der Wissenschaften der DDR, Mohrenstrasse 39, 108 Berlin, German Democratic Republic 